\title{
A Novel Preprocessing Tool to Enhance ECG R-wave Extraction
}

\author{
Sasan Yazdani*, Jean-Marc Vesin \\ Applied Signal Processing Group, Swiss Federal Institute of Technology, Lausanne, Switzerland
}

\begin{abstract}
Various approaches have been proposed to detect the $R$-waves in the ECG. From the derivative-based to more complicated wavelet transform methods, the main goal of these approaches is to extract the $R$-waves from the perturbations present in the ECG. Our study aims at proposing a simple preprocessing tool that suppresses perturbations and enhances the R-waves in the ECG. Using sliding windows, short- and long-term signal energies are calculated for each sample in the ECG. A coefficient signal is then created as the ratio between the corresponding shortand long-term energies. The enhanced ECG is then calculated by multiplying the coefficient signal and the original ECG. The MIT-BIH database was used for evaluation and the proposed method was tested against synthetic white and EMG noises. Using the proposed method as a preprocessing tool to the classic Pan-Tompkins approach lead to a significant decrease over the number of false positive and false negative QRS complexes, when synthetic noise is added to ECG.
\end{abstract}

\section{Introduction}

Electrical activity within the heart generated in the atria and propagated throughout the heart can be captured at body surface. A time-series representation of these activity, i.e. the electrocardiogram (ECG), comprises different waveforms representing depolarization and repolarization of different sections of the heart. Detection of heartbeats brings useful information that enables studies such as heart rate variability (HRV), cardiac disease diagnostics and the detection of ectopic beats such as premature ventricular contractions (PVC). The QRS complex is the most distinct waveform in the ECG. It corresponds to the contraction of ventricles, and has been mainly used for automatic heartbeat detection. However, the detection of R-waves is not an easy task as perturbations such as power-line interference and base-line wandering can be present in the ECG.

Over the years many R-wave detection algorithms have been proposed. A review of traditional approaches can be found in [1][2]. From basic derivative to complex approaches such as time-frequency analysis, generally the
ECG at hand is first filtered by either low-pass filtering in order to remove high frequency activities and remove the effect of power-line interference, or by band-pass filtering to remove the effect of baseline wandering as well as the aforementioned unwanted activities. Then, the filtered ECG is further analyzed in order to extract the Rwaves using heuristically chosen features. Pan and Tompkins proposed a detection approach based on the analysis of slope, amplitude and width of the QRS-complexes [3]. Mathematical morphology methods benefit from the shape and location of fiducial points in the QRS-complex and used them to extract R-waves [4][5]. Filter bank and Wavelet methods detect QRS-complexes by investigating modulus maxima in respectively different sub-bands and wavelet scales [6][7].

All QRS complex detection methods aim at finding a suitable mathematical description which can identify the R-wave in the ECG while suppressing perturbations and other ECG waveforms, i.e. P-,T- and U-waves. While complex methods have proven to be powerful R-wave detectors, they are not suitable for low cost and energy consumption scenarios such as body area networks (BAN). In BANs computation cost and power consumption set the limits and therefore fast, energy efficient and yet robust methods are only considered as suitable candidates. In this paper, we aim at proposing a novel, fast and efficient algorithm that can be used as an R-wave detection method or as a preprocessing tool to other R-wave detection methods. The proposed method works based on the relative shortand long-term energies in the ECG and aims at suppressing perturbation while enhancing ECG R-waves.

\section{Methods}

\subsection{Evaluation Data}

In order to evaluate the proposed method, the publicly available database of MIT/BIH-arrhythmia database was used [8]. Over the years, this database has become a standard database on which several algorithms have reported their results. The MIT/BIH arrhythmia database has a set of 48 two-lead ECG recordings with a length of 30 minutes with a sampling frequency of $360 \mathrm{~Hz}$ and 11-bit resolution within the range of $10 \mathrm{mv}$. The evaluation on this database 


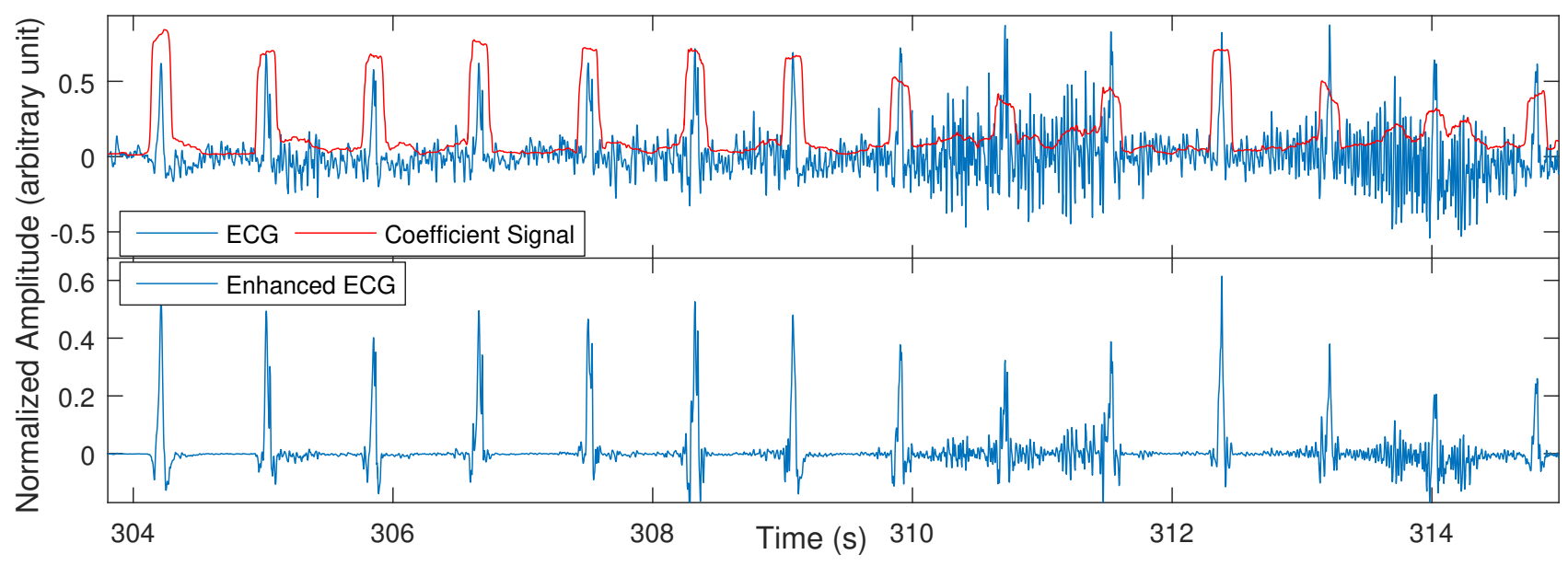

Figure 1. Performance of the relative short-long term energy on Tape 104 of the MIT/BHT arrhythmia database.

was performed on the first lead, which is either a modified lead II or lead V5, and results were checked with the reference annotation file provided for this database.

\subsection{Method Outline}

By analyzing the QRS complex in noisy ECG recordings, we came to the understanding that even in cases where severe levels of noise are presents in the ECG, QRS complexes are discernible due to the impulsiveness of Rwaves. In other words there is a meaningful difference between the baseline power and that of a QRS complex. The proposed method in this paper, referred to as the relative energy algorithm, works on this basis.

Using two sliding windows, short- and long-term signal energy powers are calculated for each sample in the ECG. For each sample a coefficient signal is created using Eq. 1.

$$
\operatorname{Coeff}(n)=\frac{\sum_{i=n-s_{w i n}}^{n+s_{w i n}}(\operatorname{Sig}(i))^{2}}{\sum_{j=n-l_{w i n}}^{n+l_{w i n}}(\operatorname{Sig}(j))^{2}}
$$

Where $n$ represents the $n^{\text {th }}$ sample of Sig. Parameters $s_{\text {win }}$ and $l_{\text {win }}$ are respectively half of the length of short and long sliding windows.

In our study, the short sliding window has a duration of $150 \mathrm{~ms}$ while the longer sliding window has a one second duration. Afterwards, the non-negative coefficient signal is divided by its maximum value in order for it to have a range of $[0,1]$. Finally, the enhanced ECG is calculated by multiplying the coefficient signal and the original ECG. As QRS complexes have relatively higher energy in comparison with P-, T-waves and the noise in the signal, the coefficient signal values are close to one where QRS complexes take place in the ECG while smaller elsewhere. Fig.1 illustrates an example of ECG enhancement based on the short-long term relative energy on a noisy part of tape 104 of the MIT/BIH arrhythmia database.

\section{Results and discussion}

In order to evaluate the proposed algorithm on the MIT/BIH arrhythmia database, first ECGs were high-pass filtered with a $4 \mathrm{~Hz}$ cutoff frequency followed by applying the relative energy algorithm. Then, the R-waves were extracted from the output by finding peaks with normalized amplitude greater than 0.02 and a minimum distance of $250 \mathrm{~ms}$. Tape-by-tape results are reported in table 1 . In this table, the detection error rate (DER) as well as sensitivity (Se) and positive prediction value (PPV), calculated through Eq. 2-4, were used for evaluation.

$$
\begin{gathered}
S e=\frac{T P}{T P+F N} \\
P P V=\frac{T P}{T P+F P} \\
D E R=\frac{F P+F N}{\text { Total No.of Beats }}
\end{gathered}
$$

where TP, FN and FP respectively represent true positive, false negative, and false positive beats calculated based on the reference annotation file.

Table 2 compares the performance obtained by our algorithm with that of well-known QRS detection methods. As shown in this table, the relative energy algorithm leads to robust ECG R-wave extraction with results comparable to that of the state-of-the art.

Results reported in tables 1 and 2, suggest that the proposed method can efficiently extract $\mathrm{R}$-waves from the ECG. In order to find out how the proposed method performs when perturbations are present the ECG, we evaluated it against white and synthetic electromyographic 
Table 1. Performance of the proposed method on QRS complex detection on MIT/BIH arrhythmia database.

\begin{tabular}{|c|c|c|c|c|c|c|}
\hline Tape No. & No. of Beats & FP & FN & DER \% & Sensitivity & PPV \\
\hline$\overline{100}$ & 2272 & 0 & 0 & $\overline{0}$ & $\overline{11}$ & 1 \\
\hline 101 & 1869 & 2 & 2 & 0.11 & 0.9989 & 0.9989 \\
\hline 102 & 2186 & 0 & 0 & 0 & 1 & 1 \\
\hline 103 & 2083 & 0 & 0 & 0 & 1 & 1 \\
\hline 104 & 2228 & 28 & 0 & 0 & 1 & 0.9874 \\
\hline 105 & 2602 & 23 & 14 & 0.54 & 0.9946 & 0.9911 \\
\hline 106 & 2027 & 1 & 0 & 0 & 1 & 0.9995 \\
\hline 107 & 2137 & 0 & 0 & 0 & 1 & 1 \\
\hline 108 & 1771 & 20 & 1 & 0.056 & 0.9994 & 0.9887 \\
\hline 109 & 2531 & 0 & 0 & 0 & 1 & 1 \\
\hline 111 & 2124 & 2 & 1 & 0.047 & 0.9995 & 0.9991 \\
\hline 112 & 2538 & 0 & 0 & 0 & 1 & 1 \\
\hline 113 & 1794 & 0 & 0 & 0 & 1 & 1 \\
\hline 114 & 1880 & 1 & 0 & 0 & 1 & 0.9995 \\
\hline 115 & 1958 & 0 & 1 & 0.051 & 0.9995 & 1 \\
\hline 116 & 2411 & 1 & 17 & 0.71 & 0.9929 & 0.9996 \\
\hline 117 & 1534 & 1 & 0 & 0 & 1 & 0.9993 \\
\hline 118 & 2278 & 1 & 0 & 0 & 1 & 0.9996 \\
\hline 119 & 1987 & 0 & 0 & 0 & 1 & 1 \\
\hline 121 & 1862 & 0 & 0 & 0 & 1 & 1 \\
\hline 122 & 2477 & 0 & 2 & 0.081 & 0.9992 & 1 \\
\hline 123 & 1518 & 0 & 0 & 0 & 1 & 1 \\
\hline 124 & 1618 & 1 & 0 & 0 & 1 & 0.9994 \\
\hline 200 & 2600 & 8 & 2 & 0.077 & 0.9992 & 0.9969 \\
\hline 201 & 1963 & 0 & 6 & 0.31 & 0.9969 & 1 \\
\hline 202 & 2137 & 2 & 3 & 0.14 & 0.9986 & 0.9991 \\
\hline 203 & 3006 & 7 & 38 & 1.3 & 0.9873 & 0.9976 \\
\hline 205 & 2656 & 0 & 5 & 0.19 & 0.9981 & 1 \\
\hline 207 & 2334 & 7 & 1 & 0.043 & 0.9996 & 0.997 \\
\hline 208 & 2962 & 1 & 20 & 0.68 & 0.9932 & 0.9997 \\
\hline 209 & 3011 & 2 & 0 & 0 & 1 & 0.9993 \\
\hline 210 & 2650 & 6 & 10 & 0.38 & 0.9962 & 0.9977 \\
\hline 212 & 2748 & 1 & 0 & 0 & 1 & 0.9996 \\
\hline 213 & 3250 & 0 & 1 & 0.031 & 0.9997 & 1 \\
\hline 214 & 2266 & 2 & 4 & 0.18 & 0.9982 & 0.9991 \\
\hline 215 & 3362 & 0 & 1 & 0.03 & 0.9997 & 1 \\
\hline 217 & 2209 & 0 & 1 & 0.045 & 0.9995 & 1 \\
\hline 219 & 2154 & 0 & 0 & 0 & 1 & 1 \\
\hline 220 & 2047 & 0 & 0 & 0 & 1 & 1 \\
\hline 221 & 2427 & 0 & 3 & 0.12 & 0.9988 & 1 \\
\hline 222 & 2482 & 2 & 0 & 0 & 1 & 0.9992 \\
\hline 223 & 2604 & 0 & 0 & 0 & 1 & 1 \\
\hline 228 & 2077 & 24 & 6 & 0.29 & 0.9971 & 0.9884 \\
\hline 230 & 2257 & 0 & 0 & 0 & 1 & 1 \\
\hline 231 & 1570 & 0 & 0 & 0 & 1 & 1 \\
\hline 232 & 1780 & 16 & 0 & 0 & 1 & 0.991 \\
\hline 233 & 3080 & 0 & 4 & 0.13 & 0.9987 & 1 \\
\hline 234 & 2753 & 0 & 1 & 0.036 & 0.9996 & 1 \\
\hline Total & 110070 & 159 & 144 & 0.28 & 0.9987 & 0.9986 \\
\hline
\end{tabular}

Table 2. Comparison of performance with previously proposed methods on MIT/BIH arrhythmia database.

\begin{tabular}{|c|c|c|c|c|c|}
\hline Fiducial point & No. of Beats & FP & FN & Failed detection \% & Ref. No. \\
\hline \hline REL_EN & 110070 & 159 & 144 & 0.28 & - \\
\hline Pan and Tompkins & 116137 & 507 & 277 & 0.675 & {$[3]$} \\
\hline Li et al. & 104184 & 65 & 112 & 0.170 & {$[7]$} \\
\hline Yazdani and Vesin & 109494 & 108 & 137 & 0.224 & {$[4]$} \\
\hline Zhang and Lian & 109510 & 204 & 213 & 0.38 & {$[9]$} \\
\hline Ravanshad et al. & 109428 & 651 & 1216 & 1.71 & {$[10]$} \\
\hline Martinez et al. & 109428 & 153 & 220 & 0.34 & {$[11]$} \\
\hline Bahoura et al. & 109809 & 135 & 184 & 0.29 & {$[12]$} \\
\hline Moody and Mark & 109428 & 94 & 1861 & 1.79 & {$[13]$} \\
\hline Lee et al. & 109481 & 137 & 135 & 0.43 & {$[14]$} \\
\hline
\end{tabular}
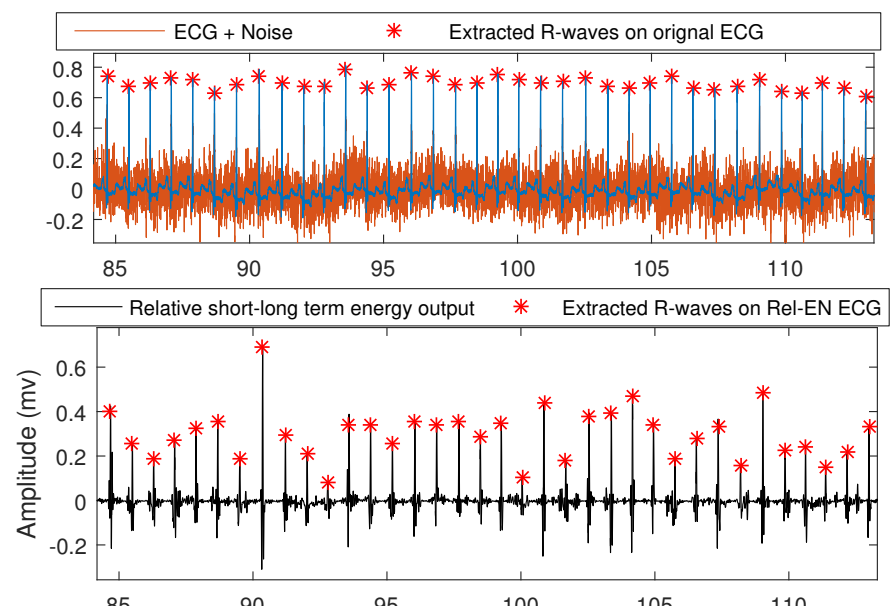

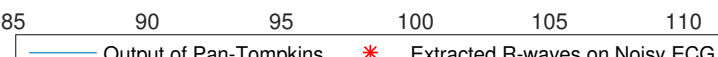

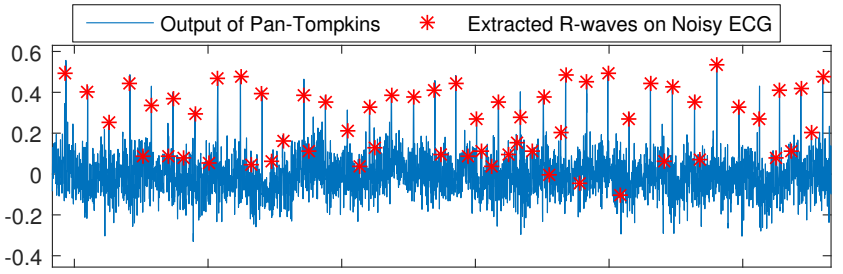

$-0.4$

$\begin{array}{llllll}85 & 90 & 95 & 100 & 105 & 110\end{array}$

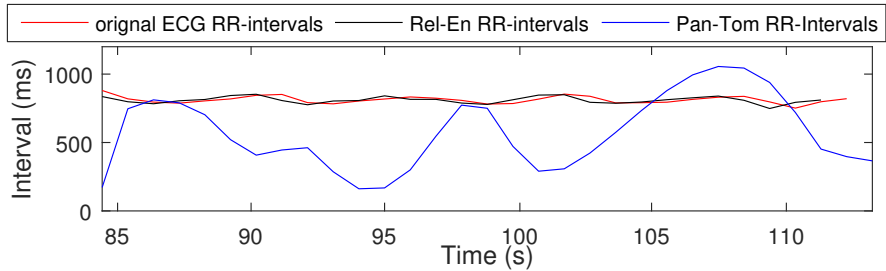

Figure 2. Performance of the relative short-long term energy against added synthesized EMG noise. RR-intervals are uniformly sampled in time, for demonstration. Tape 100 of the MIT/BHT arrhythmia database.

(EMG)noise, created by fitting an autoregressive model on EMG recordings from the Physionet/CinC2014 challenge database [15]. Using clean segments of ECGs in the MIT/BIH arrhythmia database, different levels of noise, from an input signal-to-noise ratio (SNR) of 100 to 20, were incrementally added to the ECG. Then, R-wave extraction performance was tested against Pan-Tompkins algorithm and the hybrid Pan-Tompkins/relative shortlongterm energy algorithm, in which the output of the proposed method was used as the input to the Pan-Tompkins QRS detection algorithm. Results show robust extraction of R-waves up to an input SNR of 0 or below, both against white and synthetic EMG noises. Figure 2 illustrates the performance of the hybrid algorithm and compares it with Pan-Tompkins. In this example, an EMG noise was added to tape 100 from the MIT/BIH arrhythmia database making an input SNR of -0.12. Since the number of extracted $\mathrm{R}$-waves are different in the top three sub-figures, the cal- 
culated RR-intervals were uniformly sampled in time in order to have a more sensible comparison. As seen in this figure, the RR-interval extraction is significantly improved when the hybrid approach is applied to the ECG, with similar results on white noise. This simply defined algorithm can be used to make R-wave extraction more robust.

\section{Conclusion}

In this paper, we propose a simple preprocessing tool that uses the ratio between short- and long-term ECG energies in order to suppress perturbations and make QRS complexes more prominent in the ECG. The proposed method is simply defined and can be easily computed, making it a suitable preprocessing tool for QRS complex detection algorithms. Using two simple thresholds on the output of the proposed method, an efficient R-wave extractor was created and evaluated on the MIT/BIH arrhythmia database. With sensitivity of $99.87 \%$ and a detection error rate of $0.28 \%$, the proposed method obtained results comparable to more complex state-of-the-art algorithms.

\section{Acknowledgements}

This study was performed in the framework of the NanoTera initiative supported by the Swiss National Science Foundation (SNSF).

\section{References}

[1] Kohler BU, Hennig C, Orglmeister R. The principles of software qrs detection. IEEE Magazine of Engineering in Medicine and Biology 2002;(1):42-57.

[2] Friesen GM, Jannett TC, Jadallah MA, Yates SL, Quint SR, Nagle HT. A comparison of the noise sensitivity of nine qrs detection algorithms. IEEE Transactions on Biomedical Engineering 1990;(1):85-98.

[3] Pan J, Tompkins WJ. A real-time qrs detection algorithm. IEEE Transactions on Biomedical Engineering 1985; (3):230-236. ISSN 0018-9294.

[4] Yazdani S, Vesin JM. Extraction of qrs fiducial points from the ecg using adaptive mathematical morphology. Digital Signal Processing 2016;56:100-109.
[5] Trahanias P. An approach to qrs complex detection using mathematical morphology. IEEE Transactions on Biomedical Engineering 1993;(2):201-205. ISSN 0018-9294.

[6] Dokur Z, Ölmez T, Yazgan E, Ersoy OK. Detection of ecg waveforms by neural networks. Medical engineering physics 1997;19(8):738-741.

[7] Li C, Zheng C, Tai C. Detection of ecg characteristic points using wavelet transforms. IEEE Transactions on Biomedical Engineering 1995;(1):21-28. ISSN 0018-9294.

[8] Moody GB, Mark RG. The impact of the mit-bih arrhythmia database. IEEE Magazine of Engineering in Medicine and Biology 2001;20(3):45-50.

[9] Zhang F, Lian Y. Qrs detection based on multiscale mathematical morphology for wearable ecg devices in body area networks. IEEE Transactions on Biomedical Circuits and Systems 2009;(4):220-228. ISSN 1932-4545.

[10] Ravanshad N, Rezaee-Dehsorkh H, Lotfi R, Lian Y. A level-crossing based qrs-detection algorithm for wearable ecg sensors. IEEE Journal of Biomedical and Health Informatics 2014;18(1):183-192. ISSN 2168-2194.

[11] Martínez JP, Almeida R, Olmos S, Rocha AP, Laguna P. A wavelet-based ecg delineator: evaluation on standard databases. IEEE Transactions on Biomedical Engineering 2004;51(4):570-581.

[12] Bahoura M, Hassani M, Hubin M. Dsp implementation of wavelet transform for real time ecg wave forms detection and heart rate analysis. Computer methods and programs in biomedicine 1997;52(1):35-44.

[13] Moody GB, Mark RG. Development and evaluation of a 2lead ecg analysis program. Computers in cardiology 1982; 9:39-44.

[14] Lee J, Jeong K, Yoon J, Lee M. A simple real-time qrs detection algorithm. In Proceedings of the 18th Annual International Conference of the IEEE, volume 4. 1996; 13961398.

[15] Moody G, Moody B, Silva I. Robust detection of heart beats in multimodal data: the physionet/computing in cardiology challenge 2014. In Computing in Cardiology 2014. IEEE, 2014; 549-552.

Address for correspondence:

Sasan Yazdan

EPFL SCI STI JMV - ELD 224 - Station 11

CH-1015 Lausanne - Switzerland.

E-mail address: sasan.yazdani@epfl.ch 